\title{
Neues zum Fähigkeitsausweis Praxislabor KHM (FAPL): E-Learning ersetzt einen von drei Kurstagen
}

\author{
Heinrich Haldi ${ }^{a}$, \\ Ueli Grüninger ${ }^{\text {, }}$ \\ Dagmar Sutz ${ }^{c}$ \\ a Präsident der Weiterbildungs- \\ kommission Praxislabor \\ des Kollegiums für Hausarzt- \\ medizin (KHM) \\ b Geschäftsführer KHM \\ c Sekretariat Praxislabor (KHM)
}

Korrespondenz:

Dr. med. Ueli Grüninger

Geschäftsführer KHM

Landhausweg 26

CH-3007 Bern

Tel. 0313700670

Fax 0313700679

khm[at]hin.ch

\section{Fähigkeitsausweis Praxislabor: warum und wozu?}

Der Fähigkeitsausweis Praxislabor KHM (FAPL) vermittelt dem Arzt das nötige Wissen, das Praxislabor zu leiten, zeitgerecht und wirtschaftlich zuverlässige Resultate zu erhalten und seine medizinischen Mitarbeiterinnen dabei kompetent zu führen.

Mit dem FAPL weist der Inhaber gegenüber den Kostenträgern (santésuisse, Kassen, Versicherungen) und letztlich gegenüber seinen Patienten nach, dass er eine hinreichende Ausbildung für das Betreiben eines Praxislabors und dessen Qualitätssicherung hat.

Allen Ärztinnen und Ärzten, die ein Praxislabor zu betreiben beabsichtigen, wird deshalb empfohlen, den Lehrgang zum Fähigkeitsausweis Praxislabor (FAPL) zu absolvieren. Der FAPL-Inhaber belegt damit seinen Einsatz für ein qualitativ hochstehendes Praxislabor und unterstützt damit den Kampf der Fachgesellschaften und Berufsverbände zur Erhaltung und Förderung des Praxislabors.

\section{Ist der Fähigkeitsausweis obligatorisch?}

Der FAPL beruht auf einer vertraglichen Verpflichtung der FMH mit der Kommission QUALAB, ist aber bis heute rechtlich gesehen noch nicht obligatorisch. Das soll sich aber in naher Zukunft ändern: Die Delegiertenversammlung der FMH hat am 9. März 2011 im Interesse des Erhalts des Praxislabors beschlossen, dem Bundesrat den Antrag zu stellen, den Besitz des Fähigkeitsausweises Praxislabor durch entsprechende Verankerung in der Krankenpflege-Leistungsverordnung KVL als obligatorisch zu erklären für all jene Fachärzte, welche Laborleistungen an die Sozialversicherungen verrechnen wollen.

\section{Für wen ist der Fähigkeitsausweis Praxislabor?}

Der Fähigkeitsausweis Praxislabor KHM richtet sich an Inhaber der folgenden Facharzttitel (oder Anwärter darauf): Allgemeine Innere Medizin (inkl. Allgemeine Medizin und Innere Medizin), Pädiatrie, Gynäkologie und Geburtshilfe, Allergologie und Immunologie, Endokrinologie und Diabetologie, Rheumatologie, Physikalische Medizin und Rehabilitation, Medizinische Onkologie, Tropenmedizin sowie praktischer Arzt.

\begin{abstract}
Zusammenfassung
Das Reglement für das Fähigkeitsprogramm Praxislabor (KHM) wurde auf 2012 bezüglich Erwerb und Rezertifizierung des Fähigkeitsausweises Praxislabor (FAPL) revidiert.

Die Neuregelung des Erwerbs betrifft nur künftige Kandidatinnen und Kandidaten (bereits erworbene FAPL behalten ihre Gültigkeit).

- Ab 2012 müssen alle Bewerber den Lehrgang für den Erwerb des FAPL absolvieren. Die seit 2001 gültige Übergangsregelung entfällt.

- Im Lehrgang für den Erwerb des FAPL KHM wird ein individuell absolvierbarer E-Learning-Teil eingeführt. Nach bestandenem Selbsttest folgt der neu auf zwei Tage verkürzte Präsenzkurs (bisher drei Tage).

- Das E-Learning-Modul als erster Teil des Lehrgangs zum Erwerb des FAPL steht auf Deutsch seit Januar 2012, auf Französisch ab 2013 allen neuen Kandidatinnen und Kandidaten zur Verfügung.
\end{abstract}

Die Rezertifizierungspflicht als zweite Änderung gilt für alle Inhaber des FAPL.

- Durch Teilnahme an der alljährlichen externen Qualitätskontrolle gemäss Konzept QUALAB wird der Fähigkeitsausweis automatisch rezertifiziert.

Die mit diesen Änderungen verbundene Revision des Fähigkeitsprogramms Praxislabor (KHM) wurde durch die Geschäftsleitung des SIWF zum 1.1.2012 in Kraft gesetzt. Weitere Informationen unter www.kollegium.ch/aus/pld.html

\section{Was ändert sich ab 2012?}

Das Reglement für den Fähigkeitsausweis Praxislabor (KHM) ist per 2012 revidiert worden. Diese Revision betrifft einerseits den Erwerb und andererseits die Rezertifizierung des Fähigkeitsausweises.

Die Neuregelung des Erwerbs betrifft nur künftige Kandidatinnen und Kandidaten (bereits erworbene FAPL behalten ihre Gültigkeit):

- Ab 2012 müssen alle Bewerber den Lehrgang für den Erwerb des FAPL absolvieren. Die seit 2001 
Was vermittelt der Lehrgang Praxislabor?

Dies sind die Hauptlernziele des Lehrgangs Fähigkeitsausweis Praxislabor (KHM):

- Der Arzt hat das nötige Wissen, das Praxislabor zu leiten, zeitgerecht und wirtschaftlich zuverlässige Resultate zu erhalten und seine medizinischen Mitarbeiterinnen dabei kompetent zu führen.

- Er kennt die wichtigsten Methoden und Apparate mit ihren Grenzen und kann neue Geräte oder Tests für sein Praxislabor evaluieren.

- Er kennt die Prinzipien der internen und externen Qualitätskontrolle und kann deren Resultate selbst beurteilen.

- Er kennt die häufigsten Fehlerquellen der Analytik, kann die verschiedenen Fehlerarten in der Qualitätskontrolle erkennen und so seine Praxisassistentin oder Laborantin bei der Fehlerbehebung beraten.

- Er kennt die Voraussetzungen für eine sichere, korrekte Entnahme von Körperflüssigkeiten und die Fehler der Präanalytik und kann die Qualität der Proben beurteilen.

- Er kennt die gesetzlichen Grundlagen zum Führen eines Praxislabors. gültige Übergangsregelung entfällt, wonach der Fähigkeitsausweis auch ohne Kurs erteilt werden konnte, falls ein Bewerber seinen Facharzttitel vor Ende 2002 erworben oder schon ein Praxislabor geführt hatte.

- Im Lehrgang für den Erwerb des FAPL KHM wird ein individuell absolvierbarer E-Learning-Teil eingeführt. Nach bestandenem Selbsttest folgt der neu auf zwei Tage verkürzte Präsenzkurs (bisher drei Tage).

- Das E-Learning-Modul als erster Teil des Lehrgangs zum Erwerb des FAPL steht auf Deutsch seit Januar 2012 den neuen Kandidatinnen und Kandidaten zur Verfügung. Wichtig: Die französische Fassung des E-Learning-Moduls wird ab 2013 eingeführt (technische Gründe erfordern diese Etappierung).

- Durch den E-Learning-Teil kann der bisher dreitägige Präsenzkurs auf zwei Tage verkürzt werden.

- Der E-Learning-Teil vermittelt vorgängig zum praxisorientierten Präsenzkurs die nötigen theoretischen Grundlagen. Das webbasierte E-Learning-Modul kann in beliebigen Teil-Schritten individuell $\mathrm{zu}$ Hause absolviert werden und

\section{Die Neuregelung des Erwerbs betrifft nur künftige Kandidatinnen und Kandidaten.}

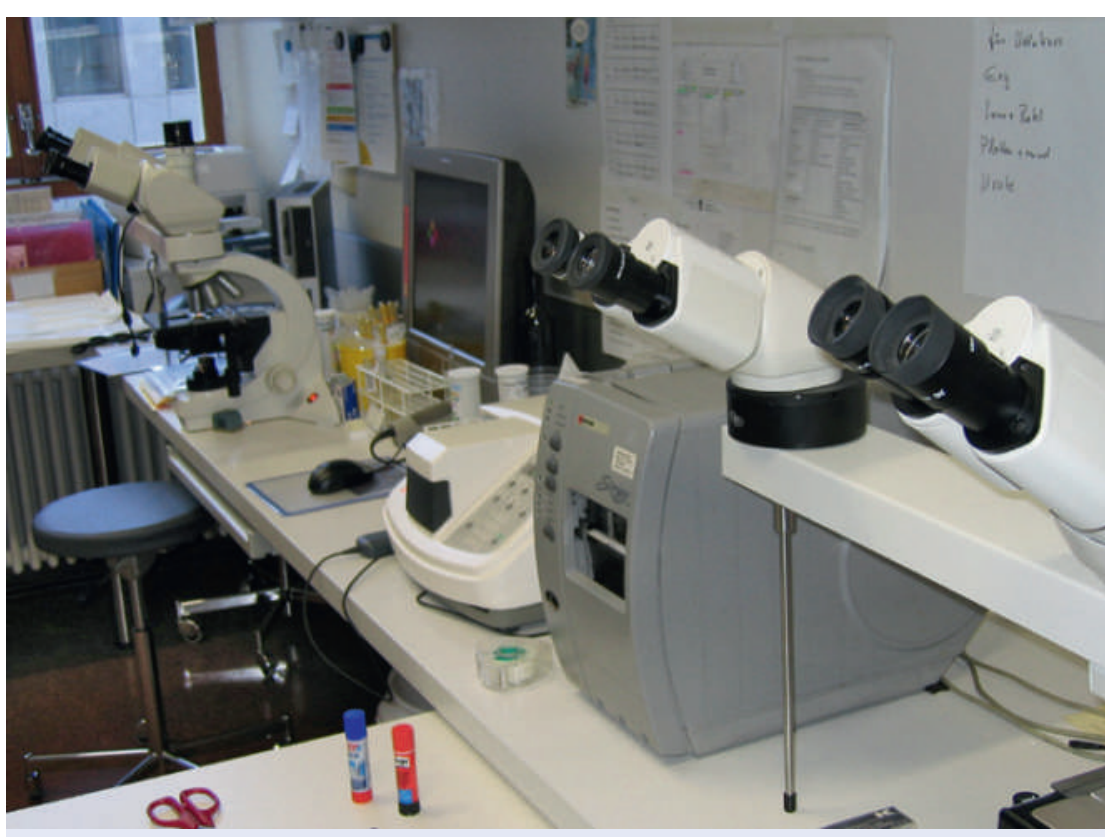

Mit dem Fähigkeitsausweis Praxislabor KHM weist der Inhaber gegenüber den Kostenträgern und Patienten nach, dass er eine hinreichende Ausbildung für das Betreiben seines Praxislabors hat. schliesst mit einem ebenfalls webbasierten Selbst-Test ab. Diese Flexibilisierung entspricht einem Wunsch vieler Teilnehmer, die dadurch weniger Arbeits- oder Frei-Tage verlieren.

- Ebenfalls neu schafft das Reglement die Möglichkeit, einen Teil der praktischen Kurszeit zu ersetzen durch entsprechende Weiterbildung am Arbeitsort (Voraussetzung: Dokumentation im Logbuch Weiterbildung der betreffenden Fachgesellschaft und Genehmigung durch die Kommission FAPL).

Eine zweite Änderung gilt für alle Inhaber des FAPL, also bisherige und neue:

- Durch die alljährliche Teilnahme an der externen Qualitätskontrolle gemäss Konzept QUALAB gilt der Fähigkeitsausweis automatisch als rezertifiziert. Diese Rezertifizierungspflicht ist neu und wird zunehmend für alle Arten von Fähigkeitsausweisen obligatorisch.

\section{Ab wann gelten die Änderungen?}

Die mit diesen Änderungen verbundene Revision des Fähigkeitsprogramms Praxislabor (KHM) wurde durch die Geschäftsleitung des Schweizerischen In- 
stituts für ärztliche Weiter- und Fortbildung (SIWF) auf 1.1.2012 in Kraft gesetzt.

\section{Wie komme ich zu einem FAPL?}

Die Schritte zum Erwerb des FAPL sind auf der Website des Kollegiums für Hausarztmedizin (KHM) detailliert dargestellt:

1. Wählen Sie dort ein Datum für den gewünschten zwei Tage dauernden praktischen Kurs: www. kollegium.ch, Rubrik AKTUELL.
6. Nach absolviertem Präsenzkurs erhalten Sie vom KHM den Fähigkeitsausweis.

Wichtig: Französischsprachige Kandidatinnen und Kandidaten absolvieren im Jahr 2012 noch den dreitägigen Präsenzkurs in Lausanne und melden sich dafür gemäss Punkt 1 und 2 oben an. Das E-Learning-Modul auf Französisch wird ab 2013 eingeführt. Ab dem Lehrgang 2013 gilt der obige Ablauf (Punkte 1-6) dann für alle.

\section{Durch die alljährliche Teilnahme an der externen Qualitätskontrolle gemäss Konzept QUALAB gilt der Fähigkeitsausweis automatisch als rezertifiziert.}

2. Melden Sie sich mit dem Download-Formular auf der Seite Praxislabor der KHM-Webseite für den gewünschten Präsenzkurs an: www. kollegium.ch/aus/pld.html

3. Sie erhalten dann die Rechnung für Lehrgang und Zertifikat, und nach Eingang der Kursgebühr bekommen Sie Anleitung und Zugangsdaten für das E-Learning-Modul Praxislabor.

4. Sie können dann das E-Learning-Modul in beliebigen Teilschritten individuell absolvieren und mit dem ebenfalls webbasierten Selbst-Test abschliessen (bis drei Versuche).

5. Sobald Sie den Test abgeschlossen haben, erhalten Sie vom KHM eine Bestätigung und die Einladung für den zweitägigen Präsenzkurs.

\section{Muss ich meinen Fachausweis Praxislabor regelmässig rezertifizieren?}

Ja; die alljährliche Teilnahme an einer externen Qualitätskontrolle gemäss QUALAB-Konzept gilt als Beleg für die Rezertifizierung. Sie brauchen also keine zusätzlichen Schritte oder Formalitäten.

\section{Informationen und Anmeldung}

Unter www.kollegium.ch/aus/pld.html sind alle Informationen detailliert dargestellt, und alle Unterlagen und das Anmeldeformular stehen zum Herunterladen bereit. Für weitere Auskünfte zum Fähigkeitsausweis Praxislabor, zu E-Learning und dem Präsenzkurs steht das Sekretariat des KHM gerne zur Verfügung (Landhausweg 26, 3007 Bern, Tel. 031 37006 70, Fax 03137006 79, e-mail: khm[at]hin.ch). 